\title{
Design and development of intelligent waste bin system with advertisement solution
}

\author{
Tarig Faisal, Moath Awawdeh, Anees Bashir
}

Department of Electrical Engineering, Higher Colleges of Technology, UAE

\begin{tabular}{l} 
Article Info \\
\hline Article history: \\
Received Nov 21, 2020 \\
Revised Jan 14, 2021 \\
Accepted Feb 6, 2021 \\
\hline
\end{tabular}

Keywords:

Advertisement solution Internet of Things Microcontroller Quality network appliance provider

Smart waste management

\begin{abstract}
In cities where a large geographical area of the city is densely populated, the process of waste collection is cumbersome, tiresome and expensive. Often, the burden of manually tracking and collecting of waste causes waste management companies enormous wasted effort and get them involved in tasks that are not necessary. No doubt, a digital interaction between waste management companies and targeted waste collection areas could ensure the process becomes fast, efficient and traceable as they become aware of the states of the wastes, aptly. It will considerably reduce any discrepancies that may occur due to the lack of information available during a particular time. Accordingly, this paper proposes a novel approach towards waste management combined with the internet of things to reduce the problems that would occur due to the accumulation of wastes and hence improvise waste collection/management process. Additionally, an innovative feature which generates revenue and creates business opportunities for waste management companies is introduced via advertisement solution based on networkattached storage technology.
\end{abstract}

This is an open access article under the CC BY-SA license.

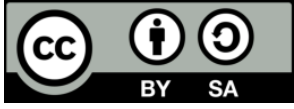

\section{Corresponding Author:}

Tarig Faisal

Department of Electrical Engineering

Higher Colleges of Technology

Ruwais, Al Dhafra, Abu Dhabi, United Arab Emirates

Email: tfaisal@hct.ac.ae

\section{INTRODUCTION}

Unarguable, urban cities across the globe are burdened with problem associated with waste management such as collection and sorting of waste. Urban cities are con- fronted with overflowing bins which cause a serious nuisance to the public. It does not only affect the overall aesthetics of the city, it is reeks of awful, lousy sight and bad smell. The foul odor can become a root cause of various diseases [1]. Broadly stated, waste management refers to various activities connected to efficient collection and effective disposal of waste; this includes many sub-activities like waste collection, waste disposal, waste transportation, and waste recycling. United Nations Department of Economic and Social Affairs statistics depicts that 3.4 billion tons of urban wastes would be generated by 2025 [2].

Prevalent waste management systems are characterized with large number of containers with obscured or inconsistent filling levels [3-4] These obscured waste bins, depending of variety of factors (such as area, location, distance, and type of waste, and even the season of the year) to get filled-up to maximum level; that too, at different and inconsistent hours, days, weeks, or even months. The type of waste bin been used makes it difficult to the waste management companies to optimally manage wastes due to the irregularities in fill-up levels which resulting in waste spillages and waste pollution. Based on the above, waste management companies and municipalities need optimal way embedded with system and tools to 
manage wastes in public places at the minimum cost and maximum efficiency. Hence, the existing waste management procedures need to be revolutionized and upgraded with the latest technology for a large-scale waste management. In recent times, there has been a widespread focus and attention on internet of things (IoT) systems embedded with cloud storage in multiple applications that create a plethora of convenient opportunities for various stakeholders for efficiently enhancing and optimizing their business procedures [5-7]. The fast-growing applications field of IoT to sensors and smart bins are already gaining attention in the waste management area, especially in urban scenarios from the point of managing resources and optimizing collecting routes [8]. Scholars have suggested expedient use of technology to improvise waste bins and ease waste collections processes. Saha et al. [9] experimented a solar panel powered compaction waste bin with mounted sensors to obtain readings of waste levels in bid to enhance the process of waste collection. The collected real-time data is transmitted wirelessly to the cloud, which can be accessed by any 3rd parties who are involved in the waste collection process. Thakker et al. [10] proposed all levels of a waste management process begin with waste collection, waste separation until, and waste disposal. In this study an alarm system triggers when the waste bin is full, and the information is relayed to the authorities when the levels of the dumps are reaching the overflow state. Near-infrared (NIR) spectroscopy was used to separate plastics from the waste and biogas plant was fed with the biodegradable wastes. Faisal et al. [11] used the developed an intelligent real-time waste segregation system that automates the process of waste segregation and classifies the waste. Real time images of the waste were used by the convolutional neural network to segregate the waste. Ramya et al. [12] proposed a system to transmit waste bins unique identity (ID) and their location to workers responsible for maintaining the bin through global system for mobile communication (GSM) whenever the levels of the waste in the container are high.

In densely populated cities, manually checking each waste bin is not only cumbersome, it is both tiresome and an ex- pensive process. Several methods were conducted to optimize waste collection and routes. Omara et al. [13] opined various parameters like maximum capacity and distribution model based on Poissons rule to estimate the arrival rate of wastes and hence improving the effectiveness of waste collection route for trucks. The system was designed in a way that an alarm is flagged off to the base station when the waste bin reaches a maximum level and then data is transmitted through the cloud to find the most effective method for collecting the waste. The system optimizes the path or route for collecting the waste as well as the operational cost. Folianto et al. [14] studied a model where the locations of dumps were identified and decided to be removed or replaced at other locations of higher demands. Using this model, the cleaning companies can plan better whether they need to sanitize the bins, clean the empty boxes with contingents, and it can also facilitate the route for the truck. Catania et al. [15] proposed a smart solution platform based on Raspberry Pi and XBee module to interconnect information and communication from various domains. The system was implemented in 2 steps; a monitoring phase where waste levels are monitored; a data processing phase for optimization of the routes for waste collection. Additionally, opportunities for residents are made available for recycling activities, by notifying them of the nearest bins. Anagnostopoulos et al. [16] exposed the decision- making process to choose between routes to maintain lost operational cost using intelligent solutions. Sensors, radio-frequency identification (RFID), and actuators were used to implement dynamic waste management systems in 3 phases: i) planning the route of waste collection, ii) transportation based on waste type, and iii) the recycling of wastes. Manqele et al. [17] introduced a decision-making algorithm for the process of waste collection. The performance of various algorithms related to the data reception, the transmission of data loss, and improving data reception were discussed. Cloud-based waste management systems were discussed in [18]. Sensors are mounted on different containers specific for different types of wastes. The obtained sensors data could be uploaded for third- party service providers to retrieve information which helps in deciding the route for waste collection.

In addition to the above system, several techniques were employed to develop smart waste pin including distribution model and machine learning $[19,20]$, and developing of models for optimal truck path for waste collection and management $[21,22]$. Those systems will be compared with our developed system in section 5.3 since they have similar feature. In line with the above studies, this research proposes a novel system that not only improvises the existing methods of waste collection but also generates revenue and creates business opportunities to waste management companies. The IoT technology is used in designing the proposed system to improve the efficiency of waste collection process which considerably reduces the associated costs. The remainder of this paper is structured as follows: the following section discusses the proposed system and its functionality. The third section presents the system operation while the circuit diagram and IoT implementation are shown in the fourth section. The hardware and software implementations are demonstrated in section five and the conclusion is drowned in the last section. 


\section{PROPOSED SYSTEM AND FUNCTIONALITY}

The main function of the proposed system to enable targeted company or municipality for better real-time monitoring and optimizing of the waste collection process by modernize and improvise the entire process of waste management. In general, the system comprises of a network of Master and Slave waste bins stations, IoT system, and advertising system based on network-attached storage technology (NAS) solutions using quality network appliance provider (QNAP) high-end product. The proposed solar-powered system is designed in the way that the Master waste bin station gathers real-time sensors data from the Slave stations and transmits them wirelessly to the IoT system. The IoT system is used to automatically visualize, analyze and export the data to a decision support system, in which a user can remotely, monitoring the waste bins network. This feature helps in real-time monitoring of the data in order to act efficiently in removing the waste and hence helps to manage waste efficiently. Additionally, this data can be accessed from any web browser or mobile device. The system is also equipped with a display screen which used for generating business opportunities for companies involved in the waste management process.

Figure 1 show the block diagram of the intelligent waste bin system which comprises of three main distinctive segments that are controlling the operation of the entire system. Setting up the Master waste bin and interconnectivity between the Master bin stations and the Slave bin stations are included in the waste bin hardware and data integration segment. In the second segment, the IoT and data management responsible of the transmission of the information and data to the IoT network. Finally, the advertisement solution segment deliberates the displaying information from the clients to the end-users via the cloud server.

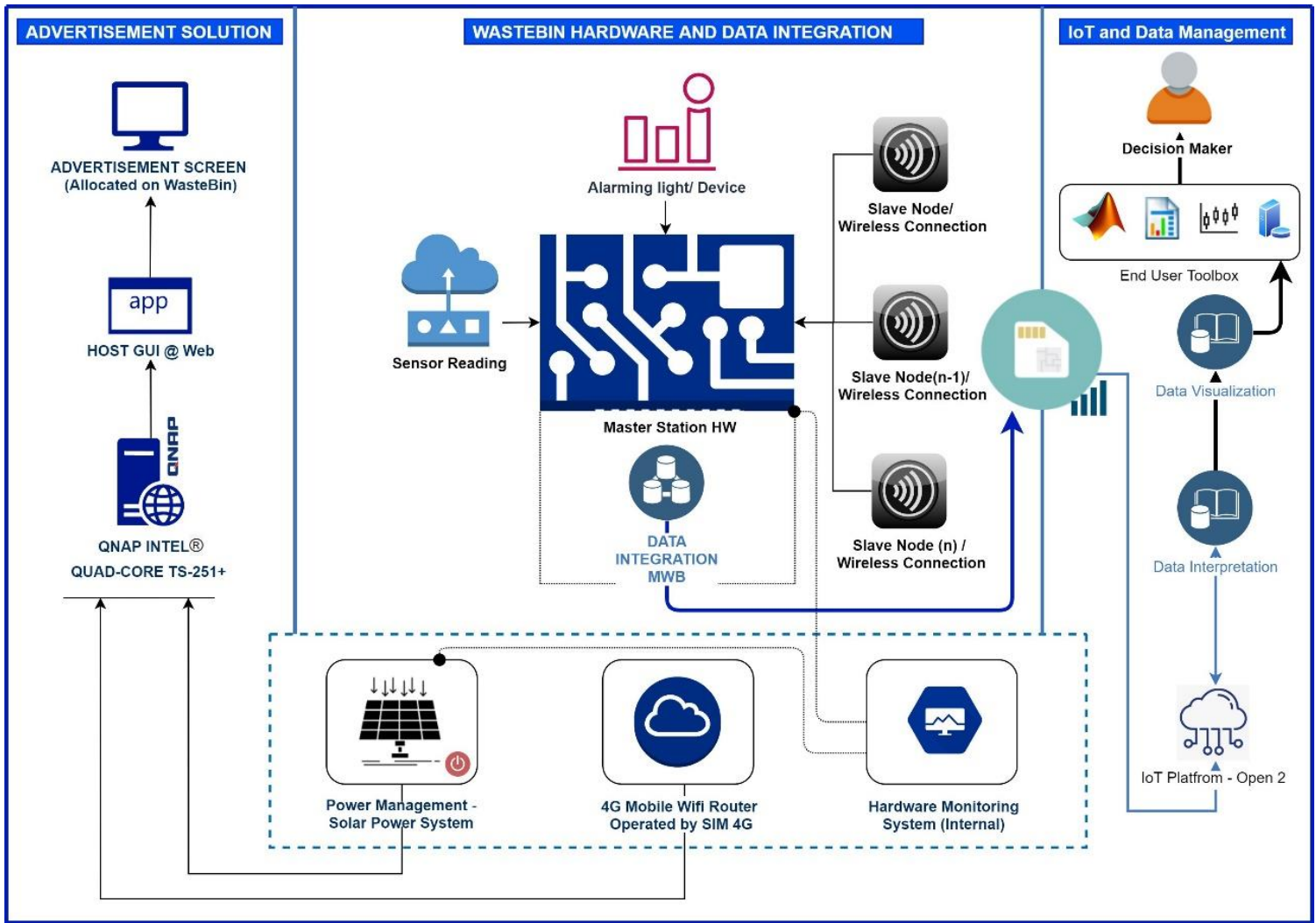

Figure 1. Block diagram of the intelligent waste bin system

\subsection{Data integration}

The section houses both the master and slave set up. It governs the overall data transformation in the entire system and the connectivity between various modules. Additionally, it forms the basis for the internet connectivity and cloud servers. The Master module is mainly involved in data extraction and processing while the Slave modules are involved in data gathering. The Slave bin stations are only responsible for collection of data via the in-house installed sensors then transmit them to the Master bin station via nodes connection. Those data comprise of various parameters including the level of waste, temperature, and gas 
detection in the system and the pin location. The extracted data is then transmitted to the IoT platform for process parameters monitoring and further data processing. The in-house sensors are also connected to three visual indicators to assist general public or users perceive the waste level by displaying different colors to indicate different measurement levels. There is also a feature of alarming protocol embedded in the system that notifies the authorities managing the process of waste collection regarding the waste levels and the presence of smoke in the waste bin.

\subsection{IoT platform and data management}

The IoT Platform is used as the main real-time data monitoring unit to process and analyze all the collected data and optimizes the resource by routing the waste collection from the control room. The control room is the main component in this system which involves in a wide variety of tasks but not limited to sharing of resources, monitoring of incurring costs, monitoring of logistics, precise location sharing, and delivering a system of high reliability and efficiency.

The task of the IoT platform begins with the Slave bin stations transmitting their measurements via their node micro-controller (NodeMCUs) to the Master bin stations NodeMCU. Then the Master station processes the data and transmits it to the IoT platform using 3G/4G link using GSM. The data is streamed via the IoT platform on a live basis and can be visualized and analyzed on a real time basis with cloud storage.

\subsection{Advertisement solution}

Since most companies depend on outdoor advertising to attract customers and consumers, the proposed advertisement solution aimed to be as a platform for those companies to reach their customers in public and therefore generating revenue for waste management companies. This is accomplished by attaching advertisement screens to the bins and paired them up with the system to display any information or advertisement. In the proposed system, the QNAP based cloud server is included as a part of the Master bin station and used as a storage source for the displayed information. The QNAP is based on NAS advanced technology which has revolutionized the field of cloud storage. It has a feature of being able to provide video control with flexibility while maintaining good security features and ease of use. It also featured with provisions for storing of multimedia and remote access that can help in providing innovative solutions to support users to develop and contribute to various IoT, artificial intelligence (AI) and machine learning solutions.

The access to the system is made from a control room through preconfigured applications connected to the web to constantly monitoring and tracking all the system parameters maintained by the QNAP cloud applications. The system users have access to the user interface via the browser using separate authentication passwords to maintain the reliability and integrity of the system. The system users display the information or advertisement via their commands which provide solutions for the management of the resources by cloud services. A separated cloud server access page is also provided for the clients to send their requested display information to the system via internet without any need of physical interaction with the system, hence it considerably reduces the maintenance cost of the system

\section{SYSTEM OPERATION AND PRINCIPLES OVERVIEW}

The interconnected-systems flowchart shown in Figure 2, illustrates the operation principles of the proposed system which contains two paths working concurrently; the main path of the data collection/processing and the standalone advertisement system. As soon as the system is started and initialized, the solar panel system is activated. Then the standalone advertisement system is activated by initializing the QNAP device, access point (via 4G router mobile), display screen, and the QNAP cloud connectivity. This part of the system is working independently from the waste bin main controller and circuitry. The main path of the data collection/processing is the main hardware/software of the waste bin; which is working as following: after the initialization of the circuit and parameters, the Master waste bin start taking the GPS reading as well as its sensors starts takes the temperature, level, and gas reading. This action is taken by running a loop namely ACT loop in which if any of the reading exceeds the pre-defined value (threshold), the alarming protocol will be activated. The activation of the alarming protocol results on sending alerting message to pre-registered number(s) and turning on the corresponding lights allocated at the waste bin. The Master waste bin is then starts collecting measurement from Slave waste bins after initializing the serial connection configuration. Once all data are collected, the Master waste bin firstly construct a matrix with all collected readings and then initiate the communication loop with Thing speak platform. The 4G module connected in the Master waste bin controller is used to send the collected data to the ThingSpeak platform for graphical representation. The ThingSpeak platform supported also with analytical tool for the decision maker where measures can be extracted also as datasets. 


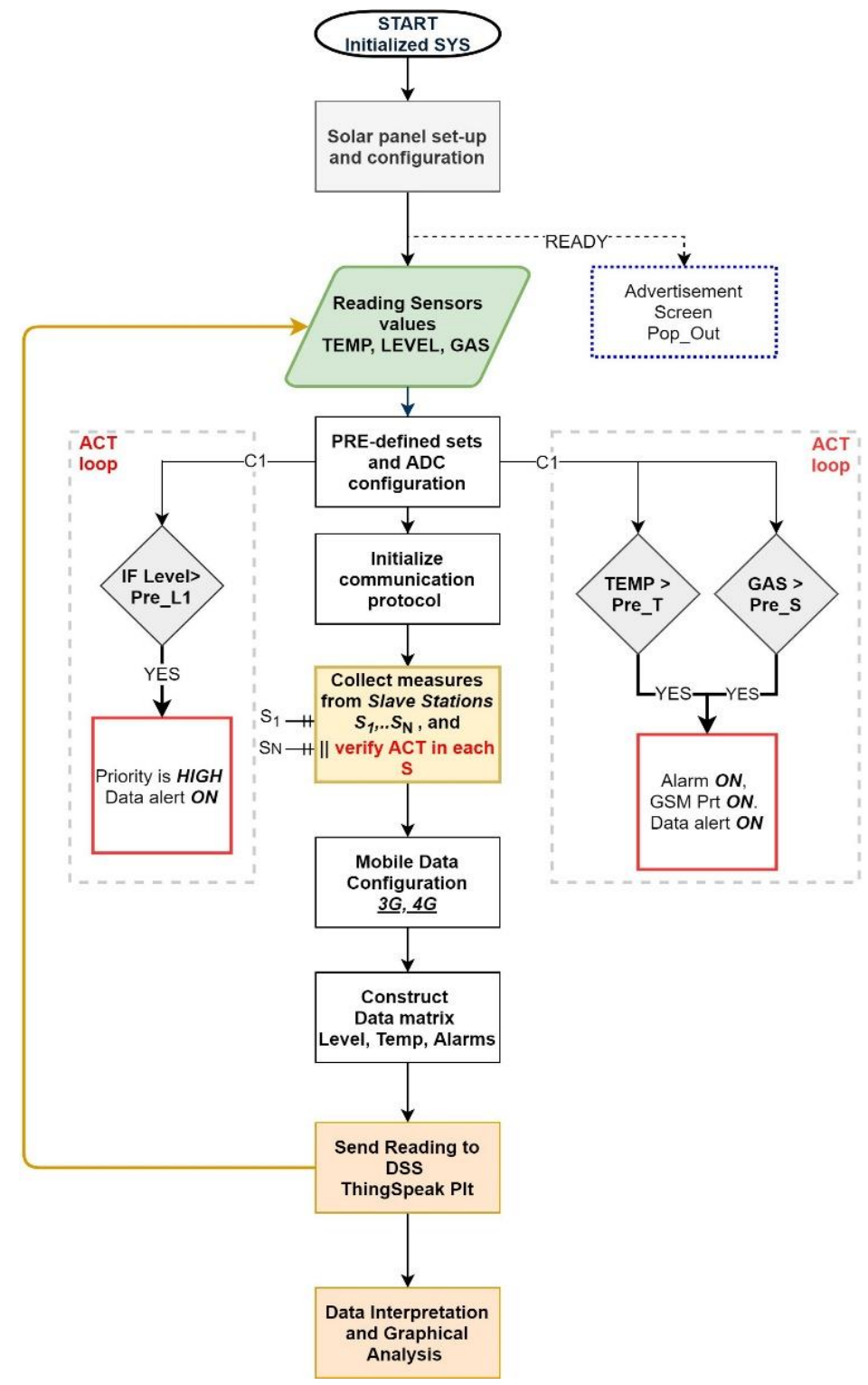

Figure 2. Flow chart of the proposed intelligent IoT waste bin system

\section{CIRCUIT DIAGRAM AND IOT IMPLEMENTATION}

The system is comprised of two circuits; the main circuit which attached to the Master station and the sub-circuit which attached to the Slave stations. The main circuit shown in Figure 3 provides full operating functionality of the system. The main supply of power constitutes of the solar panel and its peripherals. The control circuitry of the system responsible for managing and governing the entire circuit. The edge support is provided by the ATMEGA 2560 (8-bit microcontroller), which serves as the brain of the system for processes data obtained from the sensors network (level sensor, temperature, gas) and the GPS coordination. Additionally, the ATMEGA 2560 is connected to indicators to display the level of waste in the system. The green panel indicator implies that there is a low level of waste in the system, the orange panel indicator implies a medium level of occupancy, and red indicate the maximum level of waste.

The obtained data from the Slave stations is received by the NodeMCU controller and then transmit them to IoT gateway via GSM module. The GSM module is used to transmit the data the IoT platform via a 3G/4G link. The circuit also includes buck voltage converter, a real time clock module, relay module, and a voice recorder module. More details about the circuit operation can be found in [23]. The sub-circuit which attached to the Slave stations comprises of the same constituents as the Master except for the GSM module since the communication between the system and the IoT is performed only via the Master station. 


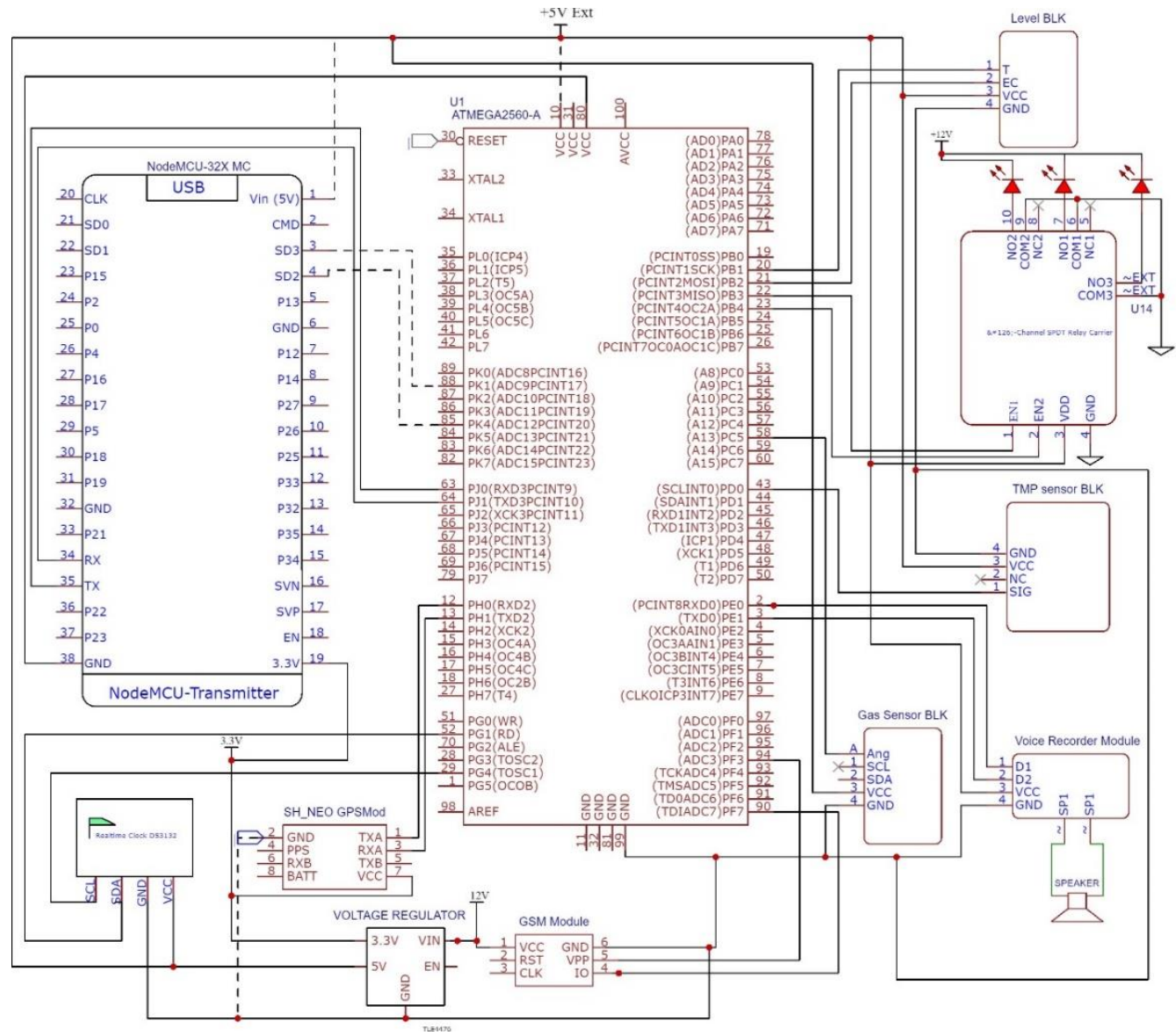

Figure 3. Circuit diagram of Master intelligent IoT waste bin system

\section{RESULTS AND DISCUSSION} sections

The hardware and software implementation of the intelligent waste bin are shown in the following

\subsection{Hardware implementation}

The power supply circuit consists of a solar panel that powers the entire circuit which produces an output voltage of around $18 \mathrm{~V} \mathrm{DC}$ to power the control circuitry and the advertisement screen. A complete solar panel circuitry is considered including a charge controller, a rechargeable DC battery, and a solar power inverter. The overall system is fitted with a digital voltmeter that indicates the level of charge stored in the battery (LED display) for monitoring the available charges percentage in the system. A buck converter board is used to steps down the incoming voltage to power various other circuitry components that require different level of voltages (including also DC-DC adjustable voltage regulator). Figure 4 shows the hardware circuitry of the system that serves as the main controller. The circuit contains the ATMEGA 2560 Arduino mega controller which serves as the data integration processor. The controller has $54 \mathrm{I} / \mathrm{O}$ pins that is used for allowing smooth connections with different peripherals such as GSM, GPS, various sensors, and relay module. The screw shields attached to the microcontroller provides robust connections between all the control circuitry. The figure also shows the NodeMCU that communicate with other Slave bin stations NodeMCUs. The Sim900a GSM module used in the circuit operates with cell phone network, controlled via AT commands with Quad-band 850-1900 MHz. A preconfigured GPS development board is used for location purposes with GPS antenna interface. The level, temperature, and gas sensor are placed underneath the circuit board. The HC SR04 transducer sensor is used to measure the level in the systems (two units are used for comparison reason). It can measure a level up to a maximum of four meters and the angle of measurement is about 15 degrees. The digital temperature sensor uses a thermistor and humidity sensor in order to monitor the surrounding environment and it takes about 2 seconds to read the real time measurement values. In order to monitor the level of gas the MQ series sensor is used which has fast response rate and good sensitivity. The voltage converters and relay board are used to control the different level of voltages in 
the circuit $(3.3,5,9$, and 12 Volts). The NodeMCU has the ESP 8266 chip with storage capacity of 4 MB external flash memory and random-access memory (RAM) of $128 \mathrm{~KB}$. The main technical challenge in hardware implantation was the communication protocol from Espino to the IoT platform from different nodes and the limitation of data format. To overcome this challenge, we have used the GSM module to send the data to ThingSpeak and creating one access point at master side to ensure mostly the data connectivity between 3 Slaves nodes and the master node.

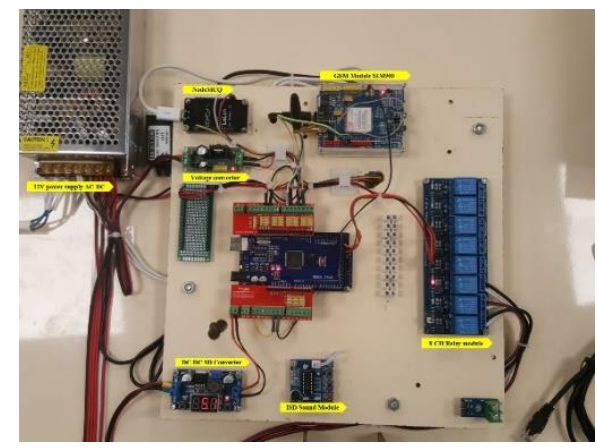

Figure 4. Main control circuitry of the intelligent waste bin

The advertisement solution is implemented using QNAP as shown in Figure 5. The basic idea of using such system is to create a remotely controlled space for sending advertisement to a connected screen. The system is mainly constructed using QNAP TS-251 plus 4TB which is high-performance NAS with onthe-fly and offline video transcoding, connected to 4G network using internet GSM 4G module of internes access point and via high-definition multimedia interface (HDMI) cable to a smart screen. Finally, the finalize prototype design is shown in Figure 6.

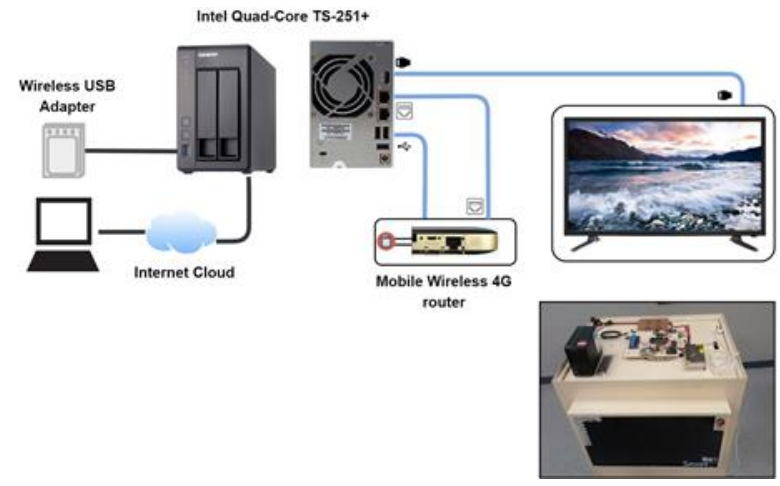

Figure 5. QNAP Connectivity and screen display

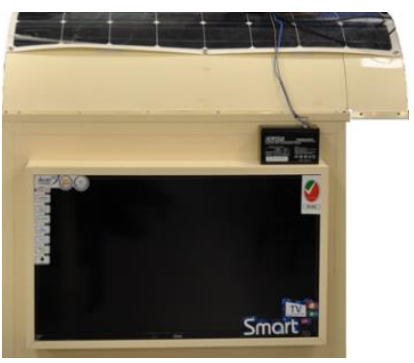

(a)

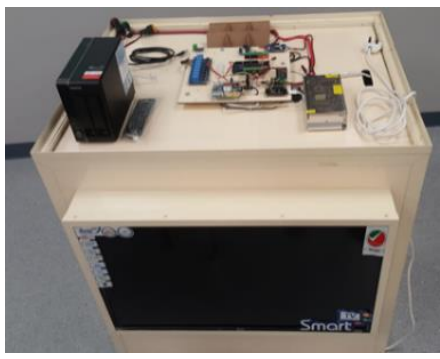

(b)

Figure 6. Final designed prototype, (a) The final prototype design with covered top, (b) The final prototype design showing control circuitry 


\subsection{Software implementation}

The system is operated with three main software platforms Arduino IDE, ThingSpeak, and QNAP cloud. Those platforms aim to serve the main circuit hardware, IoT monitoring, and advertisement solution respectively. The first platform provisions the Master station to collect the measurement from Slave waste bins. This stage requires setting up the software serial and ESP8266 Wi-Fi packages for all stations (Arduino and Espino, where NodeMCU ESP8266 has been used in many applications for remote monitoring [24, 25]. In the second platform the ThinkSpeak with academic license associated together with MATLAB license [26], is used which is widely adopted for different applications [27, 28]. The platform aims to provide generic graph representation of the received measurements over time scale for each waste bin and lamp indicator of specific waste bin whenever the level reading is exceeding $80 \%$. Figure 7 shows a sample of those graphs while Table 1, shows an example of data extraction and availability. Additionally, all available data can be exported in different format for detailed analysis using MATLAB visualization built-in tools in the project page. ThingSpeak platform provides the ability to export all channels feeds in various formats which provide an opportunity for more analysis application, and hence generating a detailed report of the waste bins network.

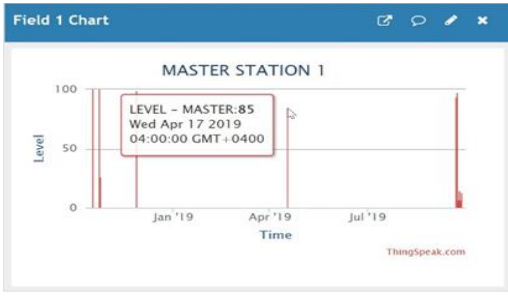

(a)

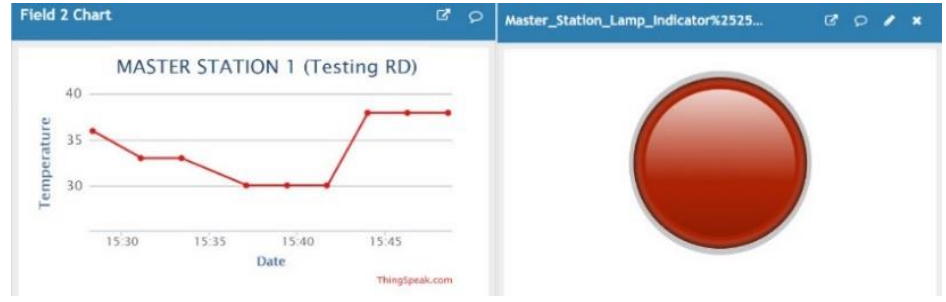

(b)

Figure 7. Sample of collected measurement, (a) Sample of level data representation, (b) Sample of temperature testing and indicator lamp

Table 1. Data extraction tools and availability

\begin{tabular}{llll}
\hline \multicolumn{1}{c}{ Tools } & \multicolumn{1}{c}{ File type } & \multicolumn{1}{c}{ Raw data } & Available index \\
\hline MATLAB visualization and analysis & .mat [m] & Level, temp, and gas \\
Export data & JSON, XML, CSV & Date and time, entity ID, field, \\
Graphical interpretation & Plot & and location for all
\end{tabular}

The proposed advertisement solution, mainly uses QNAP cloud, more specifically photo station, file station, and QTS desktop; once the connection is set-up and the module is configured, the user can upload all advertisement to the cloud in photo station sub content and hence push to show in the configured screen. MyQNAP cloud GUI shown in Figure 8, is the main environment for the end user for uploading, processing and pushing advertisement. The user needs to register for QNAP cloud and hence licensing the NAS account in order to enable the cloud remote access services.

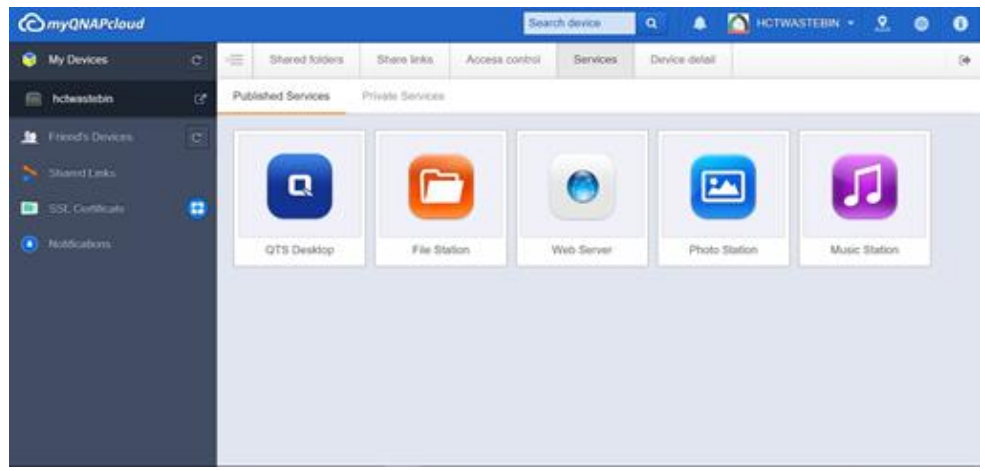

Figure 8. The main QNAP cloud page of the project 


\subsection{Comparison with existing systems}

Table 2 features the comparison between the proposed system and other some existing developed systems. In general, the proposed system contributed to the existing systems in mainly three categories: i) the design of waste bin networks which is described in previous sections as master and slave, such approach will reduce the designing cost and operational efforts, ii) the collected data and alarming flags are stored to the cloud and can be easily accessed by decision makers, iii) the advertisement solution present by using QNAP technology.

Table 2. Brief comparison of the existing smart waste bins and the proposed system

\begin{tabular}{|c|c|c|c|}
\hline Reference & Feature & Implemented HW/SW & Advantages \\
\hline $\begin{array}{l}\text { Rahman, et } \\
\text { al., } 2020 \text { [19] }\end{array}$ & $\begin{array}{l}\text { - CNN deep learning } \\
\text { - Waste classification } \\
\text { - Real-time IoT monitoring }\end{array}$ & $\begin{array}{l}\text { System is equipped with rasp-berry } \\
\text { pi, camera, sensors and Arduino for } \\
\text { the prototype with android } \\
\text { application }\end{array}$ & $\begin{array}{l}\text { - Detecting of waste level and } \\
\text { weight } \\
\text { - Classification accuracy }\end{array}$ \\
\hline $\begin{array}{l}\text { Dubey, et al., } \\
2020[20]\end{array}$ & $\begin{array}{l}\text { - KNN machine learning } \\
\text { - Waste segregation }\end{array}$ & $\begin{array}{l}\text { - System is modeled with rasp- berry } \\
\text { and Adafruit IO free web Service }\end{array}$ & $\begin{array}{l}\text { - Alarming accuracy } \\
\text { - KNN model accuracy } \\
\text { - Data visualization and dash-board }\end{array}$ \\
\hline $\begin{array}{l}\text { Khoa, et al., } \\
2020[22]\end{array}$ & $\begin{array}{l}\text { - predicting the probability of } \\
\text { the waste level in trash bins } \\
\text { - ML and graph theory } \\
\text { - LoRa technology }\end{array}$ & $\begin{array}{l}\text { - System is presented by optimal } \\
\text { algorithm combining graph theory } \\
\text { and LR (system architecture of IoT } \\
\text { and LoRa is presented) }\end{array}$ & $\begin{array}{l}\text { - System optimizes the collection } \\
\text { of waste with the shortest path } \\
\text { - low-cost design circuit } \\
\text { - System is tested in real } \\
\text { environment and saves operation } \\
\text { and time costs } \\
\text { - Mobile application }\end{array}$ \\
\hline $\begin{array}{l}\text { Omara, et al., } \\
2018[13]\end{array}$ & $\begin{array}{l}\text { - WSN-driven system } \\
\text { - ILP Model } \\
\text { - Trajectory assistant } \\
\text { - Optimal truck path }\end{array}$ & $\begin{array}{l}\text { The proposed framework is simulated } \\
\text { as the waste bins are equipped with } \\
\text { sensors for level monitoring and } \\
\text { alarms that are wirelessly } \\
\text { communicated to a cloud }\end{array}$ & $\begin{array}{l}\text { - Optimization solution } \\
\text { - Cloud platform } \\
\text { - Reduce cost of waste disposal } \\
\text { - Alarming protocol to cloud is } \\
\text { adapted }\end{array}$ \\
\hline $\begin{array}{l}\text { Proposed } \\
\text { system }\end{array}$ & $\begin{array}{l}\text { - Real-time waste monitoring } \\
\text { including temperature, level, } \\
\text { and gas monitoring } \\
\text { - Real-time authority alert } \\
\text { system } \\
\text { - Real-time advertisement } \\
\text { solution via video streaming } \\
\text { through cloud services } \\
\text { - QNAP and IoT technology }\end{array}$ & $\begin{array}{l}\text { The System is equipped with various } \\
\text { sensors, microcontrollers (ATMEGA } \\
\text { 2560), QNAP technology (QNAP } \\
\text { TS-251 plus 4TB), GSM/GPS and } \\
\text { IoT/cloud based services to collect } \\
\text { data for monitoring, analyzing, and } \\
\text { alerting in addition to podcasting } \\
\text { advertisement from the server to the } \\
\text { public. }\end{array}$ & $\begin{array}{l}\text { - Works for waste bin networks } \\
\text { - IoT End-user tool for data } \\
\text { - Analysis } \\
\text { - Advertisement solution } \\
\text { - Real-time data monitoring } \\
\text { - Cloud platform } \\
\text { - Alarming protocol } \\
\text { - Applied on real life }\end{array}$ \\
\hline
\end{tabular}

\section{CONCLUSION}

This research proposed a holistic solution aids the waste management companies and municipalities in managing wastes in public places at the minimum cost, maximum efficiency, and additional revenue. The designed smart waste bins (master and slave) combined with the IoT platform helps in facilitating real time data for easy scheduling of waste collection and route planning and hence enables the targeted company/municipality for better monitoring and optimizing the collection process. The proposed advertisement solution based on the QNAP system helps in generating extra revenue to maintain and develop the system. Additionally, the cloud server used to display information on the screen reduces the manual intervention in the system and facilitate smooth operation. From a sustainability point of view, the proposed system has the possibility of self-funding if adopted by waste management authority, thorough the revenue that would be generated via adverts. Since most organizations rely heavily on outdoor advertising to attract customers and consumers, the proposed IoT waste bin provides a veritable avenue to reach their customers at the grassroots.

\section{ACKNOWLEDGEMENTS}

This research is supported by Higher Colleges of Technology, Interdisciplinary Applied Research fund, Fund No. 103113. 


\section{REFERENCES}

[1] MS. C. Ukey, N. Bawane, and M. Mahajan, "IOT based smart garbage monitoring system," IOSR Journal of Engineering, vol. 9, no. 5, pp. 43-47, 2019.

[2] The World Bank, "Global waste to grow by 70 percent by 2050 unless urgent action is taken: world bank report," 2018. [Online]. Available at: https://www.worldbank.org/en/news/press-release/2018/09/20/global-waste-to-grow-by-70percent-by-2050-unless-urgent-action-is-taken-world-bank-report. [Accessed: 23-Nov-2020].

[3] S. V. Kumar, T. S. Kumaran, A. K. Kumar, and M. Mathapati, "Smart garbage monitoring and clearance system using Internet of Things," 2017 IEEE International Conference on Smart Technologies and Management for Computing, Communication, Controls, Energy and Materials (ICSTM), pp. 184-189, 2017.

[4] A. Borozdukhin, O. Dolinina, and V. Pechenkin, "Approach to the garbage collection in the "Smart Clean City" project," 2016 4th IEEE Int. Colloquium on Information Science and Technology (CiSt), pp. 918-922, 2016.

[5] D. Misra, G. Das, T. Chakrabortty, and D. Das, "An IoT-based waste management system monitored by cloud," Journal of Material Cycles and Waste Management, vol. 20, no. 3, pp. 1574-1582, 2018.

[6] A. A. Atayero, R. Williams, J. A. Badejo, and S. I. Popoola, "Cloud based IoT-enabled solid waste monitoring system for smart and connected communities," Int. J. of Civil Eng. and Tech., vol. 10, no. 2, pp. 2308-2315, 2019.

[7] A. S. Oliver, M. Anuradha., A. Krishnarathinam, S. Nivetha, and N. Maheswari, "IoT cloud based waste management system," Int. Conference on Computational Vision and Bio Inspired Computing, pp. 843-862, 2019.

[8] K. Pardini, J. J. P. C. Rodrigues, S. A. Kozlov, N. Kumar, and V. Furtado, "IoT-based solid waste management solutions: A survey," Journal of Sensor and Actuator Networks, vol. 8, no. 1, p. 5, 2019.

[9] H. N. Saha, S. Auddy, S. Pal, S. Kumar, S. Pandey, R. Singh, A. K. Singh, S. Banerjee, D. Ghosh, S. Saha, "Waste Management Using Internet of Things (IoT)," 2017 8th Annual Industrial Automation and Electromechanical Engineering Conference (IEMECON), pp. 359-363, 2017.

[10] S. Thakker and R. Narayanamoorthi, "Smart and wireless waste management," 2015 International Conference on Innovations in Information, Embedded and Communication Systems (ICIIECS), pp. 1-4, 2015.

[11] T. Faisal, A. Eyob, F. Debretsion, M. Tsegay, A. Bashir, and M. Awawdeh, "Development of intelligent waste segregation system based on convolutional neural network," International Journal of Advanced Science and Technology, vol. 29, no. 3, pp. 14837-14849, 2020.

[12] E. Ramya and R. Sasikumar, "A survey of smart environment conservation and protection for waste management," 2017 Third Int. Conf. on Adv. in Electrical, Elect., Infor., Comm. and Bio-Infor. (AEEICB), pp. 242-245, 2017.

[13] A. Omara, D. Gulen, B. Kantarci, and S. F. Oktug, "Trajectory-assisted municipal agent mobility: a sensor-driven smart waste management system," Journal of Sensor and Actuator Networks, vol. 7, no. 3, p. 29, 2018.

[14] F. Folianto, Y. S. Low, and W. L. Yeow, "Smartbin: smart waste management system," 2015 IEEE Tenth Int. Conference on Intelligent Sensors, Sensor Networks and Information Processing (ISSNIP), pp. 1-2, 2015.

[15] V. Catania and D. Ventura, "An approach for monitoring and smart planning of urban solid waste management using smart-M3 platform," Proc. of 15th Conference of Open Innovations Association FRUCT, pp. 24-31, 2014.

[16] T. Anagnostopoulos, A. Zaslavsky, K. Kolomvatsos, A. Medvedev, P. Amirian, J. Morley, and S. Hadjieftymiades, "Challenges and opportunities of waste management in IoT-enabled smart cities: a survey," IEEE Transactions on Sustainable Computing, vol. 2, no. 3, pp. 275-289, 2017.

[17] L. Manqele, R. Adeogun, M. Dlodlo, and L. Coetzee, "Multi-objective decision-making framework for effective waste collection in smart cities," 2017 Global Wireless Summit (GWS), pp. 155-159, 2017.

[18] M. Aazam, M. St-Hilaire, C. Lung, and I. Lambadaris, "Cloud-based smart waste management for smart cities," 2016 IEEE 21 st Int. Works. on Comp. Aided Mod. and Design of Comm. Links and Net. (CAMAD), pp. 188-193, 2016.

[19] W. Rahman, R. Islam, A. Hasan, N. I. Bithi, M. Hasan, and M. M. Rahman, "Intelligent waste management system using deep learning with IoT," Journal of King Saud University Computer and Information Sciences, 2020.

[20] S. Dubey, P. Singh, P. Yadav, and K. K. Singh, "Household waste management system using IoT and machine learning," Procedia Computer Science, vol. 167, pp. 1950-1959, 2020.

[21] E. D. Likotiko, D. Nyambo, and J. Mwangoka, "Multi-agent based IoT smart waste monitoring and collection architecture," Int. J. of Comp. Science, Eng. and Information Technology (IJCSEIT), vol. 7, no. 5, pp. 1-14, 2017.

[22] T. A. Khoa, C. H. Phuc, P. D. Lam, L. M. B. Nhu, N. M. Trong, N. T. H. Phuong, N. V. Dung, N. Tan-Y, H. N. Nguyen, and D. N. M. Duc, "Waste management system using IoT-based machine learning in university," Wireless Communications and Mobile Computing, 2020.

[23] M. Awawdeh, A. Bashir, T. Faisal, I. Ahmad, and M. K. Shahid, "IoT-based intelligent waste bin," 2019 Advances in Science and Engineering Technology International Conferences (ASET), pp. 1-6, 2019.

[24] P. Visconti, P. Visconti, N. I. Giannoccaro, R. de Fazio, S. Strazzella, and D. Cafagna, "IoT-oriented software platform applied to sensors-based farming facility with Smartphone Farmer App," Bulletin of Electrical Engineering and Informatics, vol. 9, no. 3, pp. 1095-1105, 2020.

[25] A. M. A. Jalil, R. Mohamad, N. M. Anas, M. Kassim, and S. I. Suliman, "Implementation of vehicle ventilation system using NodeMCU ESP8266 for remote monitoring," Bulletin of Electrical Engineering and Informatics, vol. 10, no. 1, pp. 327-336, 2020.

[26] "IoT Analytics-ThingSpeak Internet of Things," [Online]. Available at: https://thingspeak.com/.

[27] S. I. Abdullahi, M. H. Habaebi, and N. A. Malik, "Intelligent flood disaster warning on the fly: developing IoTbased management platform and using 2-class neural network to predict flood status," Bulletin of Electrical Engineering and Informatics, vol. 8, no. 2, pp. 706-716, 2019.

[28] A. K. Mahamad, S. Saon, H. Hashim, M. A. Ahmadon, and S. Yamaguchi, "Cloud-based people counter," Bulletin of Electrical Engineering and Informatics, vol. 9, no. 1, pp. 284-291, 2020. 